\title{
A novel nomogram predicting the overall survival of metastatic non-clear cell renal cell carcinoma patients: a large population-based investigation
}

\section{Jie Wu}

Department of Urology, National Cancer Center/National Clinical Research Center for Cancer/Cancer Hospital, Chinese Academy of Medical Sciences and Peking Union Medical College

\section{Bingqing Shang}

Department of Urology, National Cancer Center/National Clinical Research Center for Cancer/Cancer Hospital, Chinese Academy of Medical Sciences and Peking Union Medical College

\section{Ruiyang Xie}

Department of Urology, National Cancer Center/National Clinical Research Center for Cancer/Cancer Hospital, Chinese Academy of Medical Sciences and Peking Union Medical College

\section{Chuanzhen Cao}

Department of Urology, National Cancer Center/National Clinical Research Center for Cancer/Cancer Hospital, Chinese Academy of Medical Sciences and Peking Union Medical College

\section{Hongzhe Shi}

Department of Urology, National Cancer Center/National Clinical Research Center for Cancer/Cancer Hospital, Chinese Academy of Medical Sciences and Peking Union Medical College

\section{Jianzhong Shou}

Department of Urology, National Cancer Center/National Clinical Research Center for Cancer/Cancer Hospital, Chinese Academy of Medical Sciences and Peking Union Medical College

\section{Youyan Guan ( $\sim$ Guanyouyan@163.com )}

Department of Urology, National Cancer Center/National Clinical Research Center for Cancer/Cancer Hospital, Chinese Academy of Medical Sciences and Peking Union Medical College

\section{Research Article}

Keywords: metastatic renal cell carcinoma, non-clear cell renal cell carcinoma, SEER database, prognosis, nomogram

Posted Date: February 22nd, 2022

DOI: https://doi.org/10.21203/rs.3.rs-1363863/v1 
License: (c) (i) This work is licensed under a Creative Commons Attribution 4.0 International License. Read Full License 


\section{Abstract}

Objective: Metastatic non-clear cell renal cell carcinoma (non-ccmRCC) is the end stage of this disease with a poor prognosis. This study aimed to investigate prognostic clinicopathological factors and develop nomogram models for non-ccmRCC patients using a large population-based cancer database.

Methods and materials: Data of all patients with non-ccmRCC from 2010 to 2015 were retrieved from the Surveillance, Epidemiology, and End Results (SEER) database and analyzed retrospectively. Cox regression models were used to analyze prognostic risk factors for overall survival (OS). Prognostic nomogram was carried out with R software to predict the OS probability. The validation of the nomogram was performed using calibration curves and receiving operating characteristic (ROC) curve.

Results: A total of 776 non-ccmRCC patients in the SEER database were included. We separated nonccmRCC patients into three groups (group 1: one, group 2: two, group 3: three or more) according to the number of metastatic organs. Age, histology, $\mathrm{T}$ stage, $\mathrm{N}$ stage, number of metastatic organs, surgery, and chemotherapy were independent risk factors, and hence integrated into the models. The calibration curves for 6-, 12-, and 18-month OS suggested perfect agreement between the prediction by nomogram and the actual survival outcome. The 6-, 12-, and 18-month AUC of the nomogram based on the timedependent ROC curve analysis were $0.784,0.754$, and 0.766 , respectively. The result demonstrated the promising prognostic value of the nomogram.

Conclusions: We performed the first accurate nomogram for OS prediction of non-ccmRCC patients, which might help clinicians in treatment decision making.

\section{Introduction}

Renal cell carcinoma (RCC) is the third most common urologic tumor. According to statistics, of the estimated 403,262 new cases of RCC was diagnosed in 2018 worldwide ${ }^{[1]}$. Approximately $80 \%$ comprised of clear-cell renal cell carcinoma (ccRCC), while $20 \%$ were non-clear cell renal cell carcinoma (non-ccRCC) including a complex mixture of papillary RCC (pRCC), chromophobe RCC, renal medullary carcinoma (RMC), collecting duct RCC and so on in the World Health Organization (WHO) classification ${ }^{[2]}$. Most of the recent studies in RCC have restricted enrollment to patients with non-cCRCC due to small numbers of patients, without differentiating the heterogeneous types of non-ccRCC subgroups ${ }^{[3-5]}$. Despite advances in surgical treatment and medication, RCC still remains a higher risk of tumor progression and metastasis. The incidence of metastatic RCC ( $\mathrm{mRCC}$ ) is approximately $30 \%-50 \%$ during the long-term follow-up, including nearly $18 \%-30 \%$ patients with synchronous metastases at first diagnosis ${ }^{[6,7]}$. Generally, the 5 -year OS of $\mathrm{mRCC}$ reached to approximately $12.3 \%[8]$. Xue $\mathrm{J}$ et al. reported that patients of mRCC have a median overall survival (OS) for 13 months ${ }^{[9]}$. What's more, the prognosis of non-ccRCC and response to treatment have historically been inferior to $\mathrm{CCRCC}^{[10]}$. In spite of the distant metastasis, a prior study has verified that metastatic non-clear cell renal cell carcinoma (non-ccmRCC) patients could benefit from cytoreductive nephrectomy $(\mathrm{CNT})^{[11]}$. Relative to no treatment, a combination of systemic 
therapy (ST) and CNT or CNT alone or ST alone was associated survival benefits, which could be used as the treatment option for patients with non-ccmRCC ${ }^{[12]}$. As a result, earlier surveillance and intervention are much more important, as this may improve the prognosis of non-ccmRCC. However, few studies have focused on the prognosis factors for patients with non-ccmRCC.

Therefore, we carried out this research on the basis of the Surveillance, Epidemiology, and End Results (SEER) database to explore the prognosis of non-ccmRCC patients. Moreover, this study is the first nomogram to predict prognostic risk factors accurately in patients with non-ccmRCC.

\section{Materials And Methods}

\section{Data source and study population}

SEER database (http://www.seer.cancer.gov) supported by the National Cancer Institute (NCl) was used to conduct a retrospective review of patients with metastatic non-clear renal cell carcinoma, which is a cancer incidence registry that includes approximately $30 \%$ of the population of the United States. All cases were histologically confirmed as metastatic RCC (International Classification of Disease for Oncology site code C64.9) from 2010 to 2015, including papillary, chromophobe, sarcomatoid or collecting duct histological subtype. Patients were disqualified if they lacked diagnostic confirmation. Autopsy cases, bilateral or secondary tumors and negative follow-up were also excluded from analyses.

\section{Clinical and pathological variables and follow-up}

The clinical and pathological characteristics were extracted from the database, including gender, age at diagnosis, race (white, black, other), histologic subtype (papillary, chromophobe, sarcomatoid and collecting duct carcinoma), tumor size, $T$ stage in 8th edition AJCC system (T1/2/3/4 and Tx/0), N stage in 8th edition AJCC system (N0,N1,Nx), the number of metastatic organs $(1,2, \geq 3)$, surgical therapy (No surgery, Partial/Radical nephrectomy, Other/Unknown), radiotherapy (Yes, No/Unknown), chemotherapy (Yes, No/Unknown). The follow-up information contained the survival time (months) and vital survival status. The primary outcome was defined as OS. Time of OS was calculated from the diagnosis date to the death date or last contact.

\section{Statistical analysis}

SPSS V.24.0 and R version 3.6.0 were used to analyze all the data. Significant variables of $P<0.1$ in univariate Cox regression analysis were carried into multivariate analysis using the Cox proportional hazard model. On the basis of the results of the multivariable analyses $(P<0.05)$, the nomogram was developed which can provide predicted OS rates of 6,12 and 18 months with R packages ('rms' and 'survival'). Bootstraps with 1000 resamples were used for the accuracy of the nomogram. The discriminative accuracy of the nomogram was assessed based on values of the 6-, 12- and 18-month area under the receiver operating characteristic (ROC) curve. Higher AUC values between 0.5 and 1 , represent better discriminative ability of the nomogram. Besides, calibration curves were carried out to 
examine the association between the actual survival probabilities and the 6-, 12- and 18-month nomogram-predicted survival probabilities.

\section{Results}

This study encompassed 776 cases, including 558 (71.9\%) men and 218 (28.1\%) women. The demographic and clinical characteristics of patients with non-ccmRCC were presented in Table 1. Median age of all patients was 62.41 years, median tumor size was $16.32 \mathrm{~cm}$. Most harbored T3 tumors (37.2\%), N0 stage (50.8\%), and sarcomatoid histologic subtype (50.1\%). Patients of non-ccmRCC were more frequently treated with chemotherapy (58.6\%). Besides, $52.1 \%$ patients received without surgery treatment, $44.5 \%$ with CNT including partial and radical nephrectomy. Only $33.1 \%$ patients received radiotherapy. 
Table 1

Demographic and clinical characteristics of patients

\begin{tabular}{|c|c|}
\hline & Count $(n=776)$ \\
\hline \multicolumn{2}{|l|}{ Gender } \\
\hline Male & 558 (71.9) \\
\hline Female & $218(28.1)$ \\
\hline \multicolumn{2}{|l|}{ Age (year) } \\
\hline (Mean, SD) & $62.41(12.43)$ \\
\hline \multicolumn{2}{|l|}{ Race } \\
\hline White & $577(74.4)$ \\
\hline Black & $141(18.2)$ \\
\hline Other & $58(7.5)$ \\
\hline \multicolumn{2}{|l|}{ Histology } \\
\hline Papillary & $281(36.2)$ \\
\hline Chromophobe & $59(7.6)$ \\
\hline Sarcomatoid & $391(50.1)$ \\
\hline Collecting duct & $45(5.8)$ \\
\hline \multicolumn{2}{|l|}{ T stage } \\
\hline $\mathrm{T} 1$ & $145(18.7)$ \\
\hline T2 & $113(14.6)$ \\
\hline T3 & $289(37.2)$ \\
\hline $\mathrm{T} 4$ & $120(15.1)$ \\
\hline Tx/T0 & $109(14.0)$ \\
\hline \multicolumn{2}{|l|}{$\mathrm{N}$ stage } \\
\hline No & $394(50.8)$ \\
\hline N1 & $312(40.2)$ \\
\hline $\mathrm{Nx}$ & $70(9.0)$ \\
\hline \multicolumn{2}{|c|}{ Number of metastatic organs } \\
\hline 1 & 504 (64.9) \\
\hline
\end{tabular}




\begin{tabular}{|ll|}
\hline & Count $(\mathbf{n}=\mathbf{7 7 6})$ \\
\hline 2 & $192(24.7)$ \\
\hline Tumor size $(\mathrm{cm})$ & $80(10.3)$ \\
\hline (Mean, SD) & $16.32(25.62)$ \\
\hline Surgery & \\
\hline No surgery & $404(52.1)$ \\
\hline Partial/Radical nephrectomy & $345(44.5)$ \\
\hline Other/Unknown & $27(3.5)$ \\
\hline Radiotherapy & \\
\hline Yes & $257(33.1)$ \\
\hline No/Unknown & $519(66.9)$ \\
\hline Chemotherapy & \\
\hline Yes & $455(58.6)$ \\
\hline No/Unknown & $321(41.4)$ \\
\hline
\end{tabular}

Most patients occurred with single metastasis (64.9\%) and $273(35.1 \%)$ had multiple metastases at their initial diagnosis, including metastases to the lung, bone, liver, brain. In regard to the location of metastases at diagnosis, 272 (35\%) patients had lung metastasis, 150 (19.3\%) had bone metastasis, 62 (8.0\%) had liver metastasis, 20 (2.6\%) had brain metastasis. The detailed metastatic information was illustrated in Figure.1 Median follow-up was 64 months in the entire cohort, and 704 patients passed away due to tumor progression.

There was also no significant difference could be observed in OS between Bone, Brain, Liver and Lung metastatic site $(p>0.05)$ (Supplementary Figure.1). When we stratified non-ccmRCC patients into three subclasses according to the number of metastatic sites (group 1: one, group 2: two, group 3: three or more), the Kaplan-Meier analysis demonstrated that there existed extremely significant differences in OS probability according to the number of metastatic sites $(p<0.0001)$ (Supplementary Figure.2).

Finally, the univariate and multivariate analyses demonstrated that the number of metastatic organs was an independent risk factors for OS (2 metastatic sites [hazard ratio (HR) 1.432, 95\% Cl 1.200-1.709, $\mathrm{P}<$ 0.001 ] or $\geq 3$ metastatic sites [HR 1.927, 95\% Cl 1.492-2.489, $\mathrm{P}<0.001]$ ) (Table 2). Moreover, the elder [HR 1.014, 95\% Cl 1.007-1.020, P<0.001], sarcomatoid [HR 1.894, 95\% Cl 1.599-2.243, $\mathrm{P}<0.001$ ] and collecting duct subtype [HR 1.482, 95\% Cl 1.065-2.063, P = 0.020], T3/T4 [HR 1.373, 95\% Cl 1.162-1.622, $\mathrm{P}<0.001]$ and $\mathrm{N} 1$ stage [HR 1.487, 95\% Cl 1.270-1.742, $\mathrm{P}<0.001]$, partial/radical nephrectomy [HR 
$0.531,95 \% \mathrm{Cl} 0.449-0.628, \mathrm{P}<0.001]$ and chemotherapy [HR $0.675,95 \% \mathrm{Cl} 0.575-0.792, \mathrm{P}<0.001]$ treatment were independent prognostic determinants for OS (Table 2). 
Table 2

Univariate and multivariate Cox regression analysis for predicting OS

\begin{tabular}{|c|c|c|c|c|c|c|}
\hline & \multicolumn{3}{|c|}{ Univariate analysis } & \multicolumn{3}{|c|}{ Multivariate analysis } \\
\hline & $\mathrm{HR}$ & $95 \% \mathrm{Cl}$ & $p$ value & $\mathrm{HR}$ & $95 \% \mathrm{Cl}$ & $p$ value \\
\hline \multicolumn{7}{|l|}{ Gender } \\
\hline Male & Ref & & & & & \\
\hline Female & 0.996 & $0.845-1.175$ & 0.965 & & & \\
\hline Age & 1.012 & $1.006-1.018$ & $<0.001$ & 1.014 & $1.007-1.020$ & $<0.001$ \\
\hline \multicolumn{7}{|l|}{ Race } \\
\hline White & Ref & & & & & \\
\hline Black & 0.893 & $0.736-1.084$ & 0.253 & & & \\
\hline Other & 0.879 & $0.665-1.160$ & 0.361 & & & \\
\hline \multicolumn{7}{|l|}{ Histology } \\
\hline Papillary & Ref & & & Ref & & \\
\hline Chromophobe & 0.764 & $0.560-1.042$ & 0.090 & 0.885 & $0.647-1.212$ & 0.447 \\
\hline Sarcomatoid & 1.745 & $1.482-2.055$ & $<0.001$ & 1.894 & $1.599-2.243$ & $<0.001$ \\
\hline Collecting duct & 1.589 & $1.152-2.193$ & 0.005 & 1.482 & $1.065-2.063$ & 0.020 \\
\hline \multicolumn{7}{|l|}{ T stage } \\
\hline $\mathrm{T} 1 / \mathrm{T} 2$ & Ref & & & Ref & & \\
\hline $\mathrm{T} 3 / \mathrm{T} 4$ & 1.209 & $1.023-1.428$ & 0.026 & 1.373 & $1.162-1.622$ & $<0.001$ \\
\hline \multicolumn{7}{|l|}{ N stage } \\
\hline NO & Ref & & & Ref & & \\
\hline N1 & 1.418 & $1.212-1.660$ & $<0.001$ & 1.487 & $1.270-1.742$ & $<0.001$ \\
\hline \multicolumn{7}{|c|}{ Number of metastatic organs } \\
\hline 1 & Ref & & & Ref & & \\
\hline 2 & 1.582 & $1.330-1.881$ & $<0.001$ & 1.432 & $1.200-1.709$ & $<0.001$ \\
\hline$\geq 3$ & 2.111 & $1.653-2.696$ & $<0.001$ & 1.927 & $1.492-2.489$ & $<0.001$ \\
\hline
\end{tabular}

OS: overall survival; HR: hazard ratio; $\mathrm{Cl}$ : confidence interval; Ref: reference c-index:0.696 
Tumor size $(\mathrm{cm})$

$\begin{array}{llll}\leq 7 & \text { Ref } & & \\ \square 7 & 1.074 & 0.917-1.257 & 0.376\end{array}$

\section{Surgery}

No surgery

Ref

Ref

$\begin{array}{lllllll}\text { Partial/Radical nephrectomy } & 0.504 & 0.432-0.588 & <0.001 & 0.531 & 0.449-0.628 & <0.001 \\ \text { Radiotherapy } & & & & \end{array}$

No/Unknown

Ref

Yes

1.132

$0.969-1.323$

0.118

\section{Chemotherapy}

No/Unknown

Ref

Ref

Yes

0.873

0.750-1.016

0.079

0.675

$0.575-0.792$

$<0.001$

OS: overall survival; HR: hazard ratio; $\mathrm{Cl}$ : confidence interval; Ref: reference

c-index:0.696

We developed a prognostic nomogram on the basis of the results of $\mathrm{Cox} \mathrm{PH}$ regression. As illustrated in Figure.2, the length of the line corresponding to every variable in the nomogram equaled the contribution of predictors for the OS rates of 6-, 12- and 18-month. The AUC for OS rates of 6, 12 and 18 months was $0.784,0.754$ and 0.766 , respectively (Figure.3). Moreover, the 6-, 12- and 18-month calibration curves presented an excellent consistency and an acceptable consistency between nomogram predictions and actual observations (Figure.4).

\section{Discussion}

The mRCC accounts for $30 \%-50 \%$ of RCC during the long-term follow-up, including approximately $18 \%-30 \%$ patients with synchronous metastases at first diagnosis ${ }^{[6,7]}$. Therefore, it is of great importance to accurately predict the survival of mRCC patients. However, there are no large-scale contemporary studies confirming the prognostic of non-ccmRCC for the heterogeneous subgroups. Therefore, we conducted this study on the basis of the SEER database to explore the prognosis of non-ccmRCC patients including papillary, chromophobe, sarcomatoid, or collecting duct histological subtype. Due to the low incidence, this study recruited 776 patients of non-ccmRCC during 2010-2015 year in the SEER database. For non-ccmRCC in the SEER database, we identified that $64.9 \%$ of patients suffered single metastasis, and $35 \%$ of patients had multiple metastases. Lung (35\%) was the most common site of metastasis, followed by bone (19.3\%). Zheng W et al. investigated that metastases sites including bone, 
brain, liver, and lung were all significantly related to OS for metastatic RCC patients ${ }^{[13]}$. In this study, we found that the location of metastatic sites existed no significant survival difference in patients diagnosed with non-ccmRCC (Supplementary Fig. 1). When we separated non-ccmRCC patients into three groups (group 1: one, group 2: two, group 3: three or more) according to the number of metastatic organs, we identified that patients with higher number of metastatic sites showed worse survival outcomes in this cohort than those with lower numbers of metastatic organs (Supplementary Fig. 2). In Mao W et al.' prior study, for mRCC patients with lung metastasis, they identified that treatment including surgery and radiotherapy were independent risk factor of $0 S^{[14]}$. Based on their research results, we carried out this similar study with non-ccmRCC patients. We demonstrated that the higher number of metastatic sites (> 1), the elder, sarcomatoid and collecting duct subtype, T3/T4 and N1 stage were independent unfavorable prognostic determinants for OS. Similar to findings in patients with $\operatorname{mRCC}^{[14,15]}, \mathrm{T}$-stage, $\mathrm{N}$-stage and age at diagnosis were significantly associated with a poor prognosis, which may be attributed to the advanced T-stage and $\mathrm{N}$-stage related to aggressive tumor characteristic and the age-induced decline in the immune system. Notably, T3/T4 stage disease was identified as an independent prognostic factor while tumor size was not a prognostic factor, indicating that tumor invasion depth still represents poor outcome. Compared with tumor size, T-stage also represents the tumor aggressive ability of extending into veins or perinephric tissues. Collecting duct carcinoma subtype ${ }^{[16]}$ and Sarcomatoid subtype were considered as the poor prognostic risk factors for advanced RCC ${ }^{[17,18]}$. The current guideline for mRCC patients recommends that surgical treatment as CNT is beneficial and systemic treatment is required ${ }^{[19]}$. Marchioni et al have reported the effect of CNT on the survival of non-ccmRCC, relative to no treatment ${ }^{[11]}$. Besides, Luzzago et al. have identified that ST, CNT, or ST combined CNT was associated with low overall mortality ${ }^{[12]}$. Consistent with the prior studies above, our research identified a survival benefit with the treatment of CNT including partial and radical nephrectomy, or chemotherapy in nonccmRCC. In addition, we explored the prognostic role of radiotherapy and confirmed that non-ccmRCC patients could not benefit from radiotherapy. Based on these findings above, we recommended that the treatment of systemic chemotherapy and CNT could be taken into account in non-ccmRCC patients for survival benefit.

There exist the limitations of this study as followed: (1) This study was based on data from the SEER database and it was a retrospective data set. (2) This research only included 776 non-ccmRCC patient between 2010 and 2015 in the United States, which was not a large-sized study. Therefore, it is of great importance to perform multicenter clinical trials to verify the accuracy of the nomograms.

\section{Conclusions}

In this study, we first established a nomogram prognostic evaluation model for non-ccmRCC patients on the basis of the SEER database, which might help clinicians in treatment decision making.

Simultaneously, the 6-, 12- and 18-month OS nomogram model's accuracy were evaluated by ROC-curve and calibration curves. 


\section{Declarations}

Acknowledgements

Not applicable.

\section{Authors' contributions}

WJ and SBQ was responsible for data analysis and writing of the draft of the manuscript. XRY, CCZ and SHZ were responsible for data revision. SJZ and GYY reviewed/edited the manuscript. All authors contributed to the article and approved the submitted version.

\section{Funding}

This work was supported by Beijing Municipal Natural Science Foundation (ID Number: L192040).

\section{Availability of data and materials}

The datasets analyzed during the current study are available in the SEER repository (https://seer.cancer.gov/).

\section{Ethics approval and consent to participate}

Not applicable.

\section{Consent for publication}

Not applicable.

\section{Competing interests}

The authors declare that they have no conflict of interest and they have nothing to disclose.

\section{References}

1. Bray F, Ferlay J, Soerjomataram I, Siegel R L, Torre L A, Jemal A. Global cancer statistics 2018: GLOBOCAN estimates of incidence and mortality worldwide for 36 cancers in 185 countries [J]. CA Cancer J Clin, 2018, 68(6): 394-424. DOI:10.3322/caac.21492

2. Moch H, Cubilla A L, Humphrey P A, Reuter V E, Ulbright T M. The 2016 WHO Classification of Tumours of the Urinary System and Male Genital Organs-Part A: Renal, Penile, and Testicular Tumours [J]. Eur Urol, 2016, 70(1): 93-105. DOI:10.1016/j.eururo.2016.02.029

3. Lee J L, Ahn J H, Lim H Y, Park S H, Lee S H, Kim T M, et al. Multicenter phase II study of sunitinib in patients with non-clear cell renal cell carcinoma [J]. Ann Oncol, 2012, 23(8): 2108-14.

DOI:10.1093/annonc/mdr586 
4. Armstrong A J, Nixon A B, Carmack A, Yang Q, Eisen T, Stadler W M, et al. Angiokines Associated with Targeted Therapy Outcomes in Patients with Non-Clear Cell Renal Cell Carcinoma [J]. Clin Cancer Res, 2021, 27(12): 3317-28. DOI:10.1158/1078-0432.CCR-20-4504

5. McDermott D F, Lee J L, Ziobro M, Suarez C, Langiewicz P, Matveev V B, et al. Open-Label, Single-Arm, Phase II Study of Pembrolizumab Monotherapy as First-Line Therapy in Patients With Advanced Non-Clear Cell Renal Cell Carcinoma [J]. J Clin Oncol, 2021, 39(9): 1029-39. DOI:10.1200/JC0.20.02365

6. Tadayoni A, Paschall A K, Malayeri A A. Assessing lymph node status in patients with kidney cancer [J]. Transl Androl Urol, 2018, 7(5): 766-73. DOI:10.21037/tau.2018.07.19

7. Prochazkova K, Vodicka J, Fichtl J, Krakorova G, Sebek J, Rousarova M, et al. Outcomes for Patients after Resection of Pulmonary Metastases from Clear Cell Renal Cell Carcinoma: 18 Years of Experience [J]. Urol Int, 2019, 103(3): 297-302. DOI:10.1159/000502493

8. Harada K, Nozawa M, Uemura M, Tatsugami K, Osawa T, Yamana K, et al. Treatment patterns and outcomes in patients with unresectable or metastatic renal cell carcinoma in Japan [J]. Int J Urol, 2019, 26(2): 202-10. DOI:10.1111/iju.13830

9. Xue J, Chen W, Xu W, Xu Z, Li X, Qi F, et al. Patterns of distant metastases in patients with clear cell renal cell carcinoma-A population-based analysis [J]. Cancer Med, 2021, 10(1): 173-87.

DOI:10.1002/cam4.3596

10. Vera-Badillo F E, Templeton A J, Duran I, Ocana A, de Gouveia P, Aneja P, et al. Systemic therapy for non-clear cell renal cell carcinomas: a systematic review and meta-analysis [J]. Eur Urol, 2015, 67(4): 740-9. DOI:10.1016/j.eururo.2014.05.010

11. Marchioni M, Bandini M, Preisser F, Tian Z, Kapoor A, Cindolo L, et al. Survival after Cytoreductive Nephrectomy in Metastatic Non-clear Cell Renal Cell Carcinoma Patients: A Population-based Study [J]. Eur Urol Focus, 2019, 5(3): 488-96. DOI:10.1016/j.euf.2017.11.012

12. Luzzago S, Palumbo C, Rosiello G, Knipper S, Pecoraro A, Mistretta F A, et al. Association Between Systemic Therapy and/or Cytoreductive Nephrectomy and Survival in Contemporary Metastatic Nonclear Cell Renal Cell Carcinoma Patients [J]. Eur Urol Focus, 2021, 7(3): 598-607. DOI:10.1016/j.euf.2020.04.009

13. Zheng W, Zhu W, Yu S, Li K, Ding Y, Wu Q, et al. Development and validation of a nomogram to predict overall survival for patients with metastatic renal cell carcinoma [J]. BMC Cancer, 2020, 20(1): 1066. DOI:10.1186/s12885-020-07586-7

14. Mao W, Fu Z, Wang K, Wu J, Xu B, Chen M. Prognostic nomogram for patients with lung metastatic renal cell carcinoma: a SEER-based study [J]. Ann Palliat Med, 2021, 10(3): 2791-804. DOI:10.21037/apm-20-1488

15. Wang K, Wu Z, Wang G, Shi H, Xie J, Yin L, et al. Survival nomogram for patients with bone metastatic renal cell carcinoma: A population-based study [J]. Int Braz J Urol, 2021, 47(2): 333-49. DOI:10.1590/S1677-5538.IBJU.2020.0195 
16. Sheng X, Lu X, Wu J, Chen L, Cao H. A Nomogram Predicting the Prognosis of Renal Cell Carcinoma Patients with Lung Metastases [J]. Biomed Res Int, 2021, 2021 (6627562.

DOI:10.1155/2021/6627562

17. Keskin S K, Msaouel P, Hess K R, Yu K J, Matin S F, Sircar K, et al. Outcomes of Patients with Renal Cell Carcinoma and Sarcomatoid Dedifferentiation Treated with Nephrectomy and Systemic Therapies: Comparison between the Cytokine and Targeted Therapy Eras [J]. J Urol, 2017, 198(3): 530-7. DOI:10.1016/j.juro.2017.04.067

18. Zhang L, Wu B, Zha Z, Zhao H, Feng Y. The prognostic value and clinicopathological features of sarcomatoid differentiation in patients with renal cell carcinoma: a systematic review and metaanalysis [J]. Cancer Manag Res, 2018, 10(1687-703. DOI:10.2147/CMAR.S166710

19. Ljungberg B, Bensalah K, Canfield S, Dabestani S, Hofmann F, Hora M, et al. EAU guidelines on renal cell carcinoma: 2014 update [J]. Eur Urol, 2015, 67(5): 913-24. DOI:10.1016/j.eururo.2015.01.005

\section{Figures}

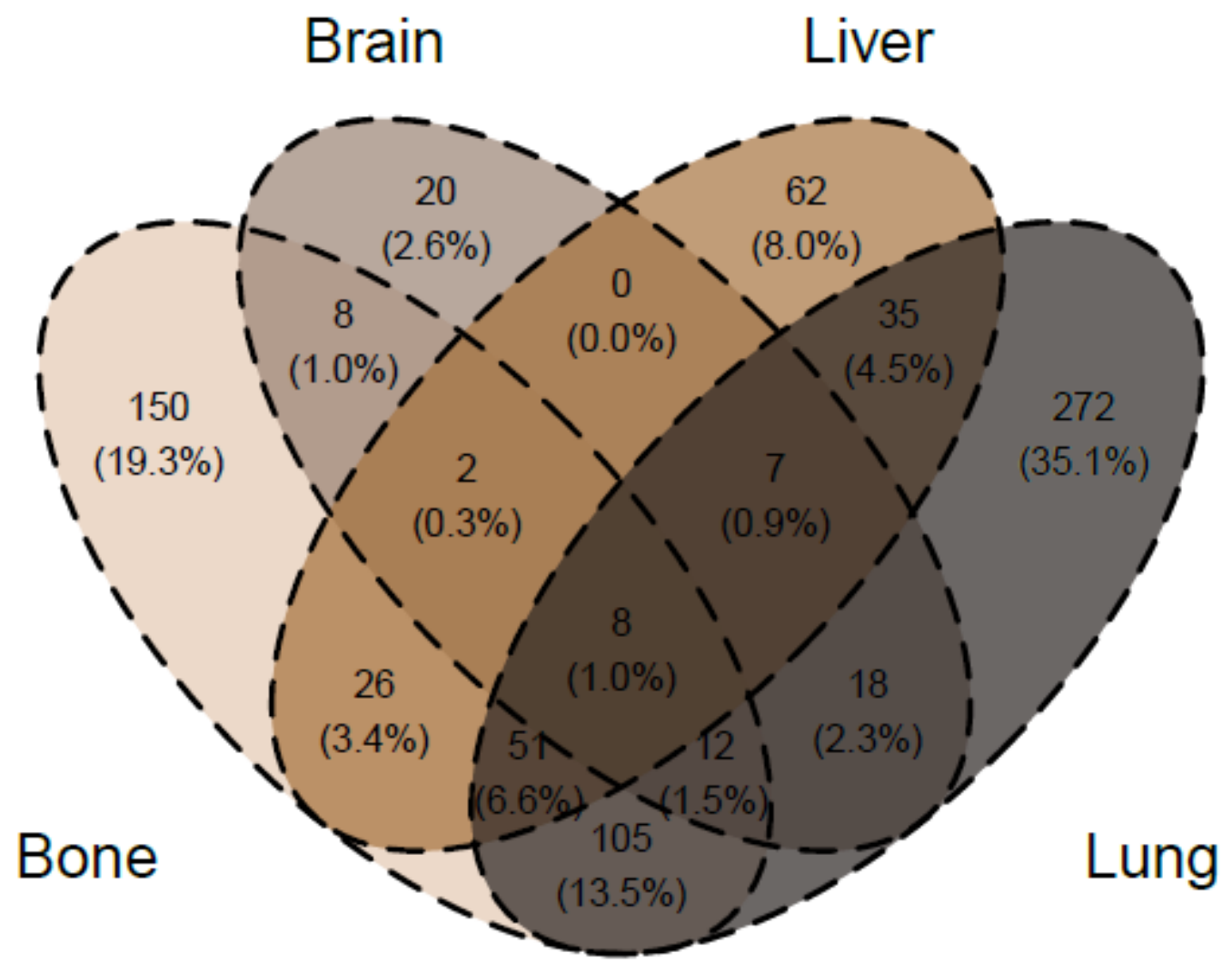

\section{Figure 1}

Venn diagram of the distribution of distant metastatic sites in the overall cohort. 
Points

age

histology

N

T

number of metastatic organs

surgery

chemotherapy

Total Points

6-month survival

12-month survival

18-month survival
0

10

20

30

40

50

60

70

80

90

100

\begin{tabular}{|c|c|c|c|c|c|c|}
\hline $\begin{array}{c}30 \\
\text { papillary }\end{array}$ & 40 & 50 & 60 & 70 & $\begin{array}{r}80 \\
\text { sarcomatoid }\end{array}$ & 90 \\
\hline
\end{tabular}

chromophobe collecting duct

yes

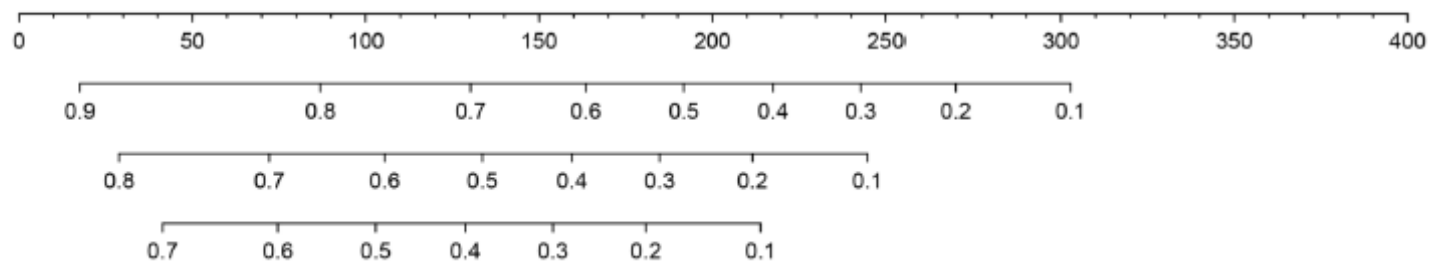

Figure 2

Nomogram predicting 6-, 12-and 18-month overall survival for patients with non-ccmRCC patients.

Time-dependent ROC for OS

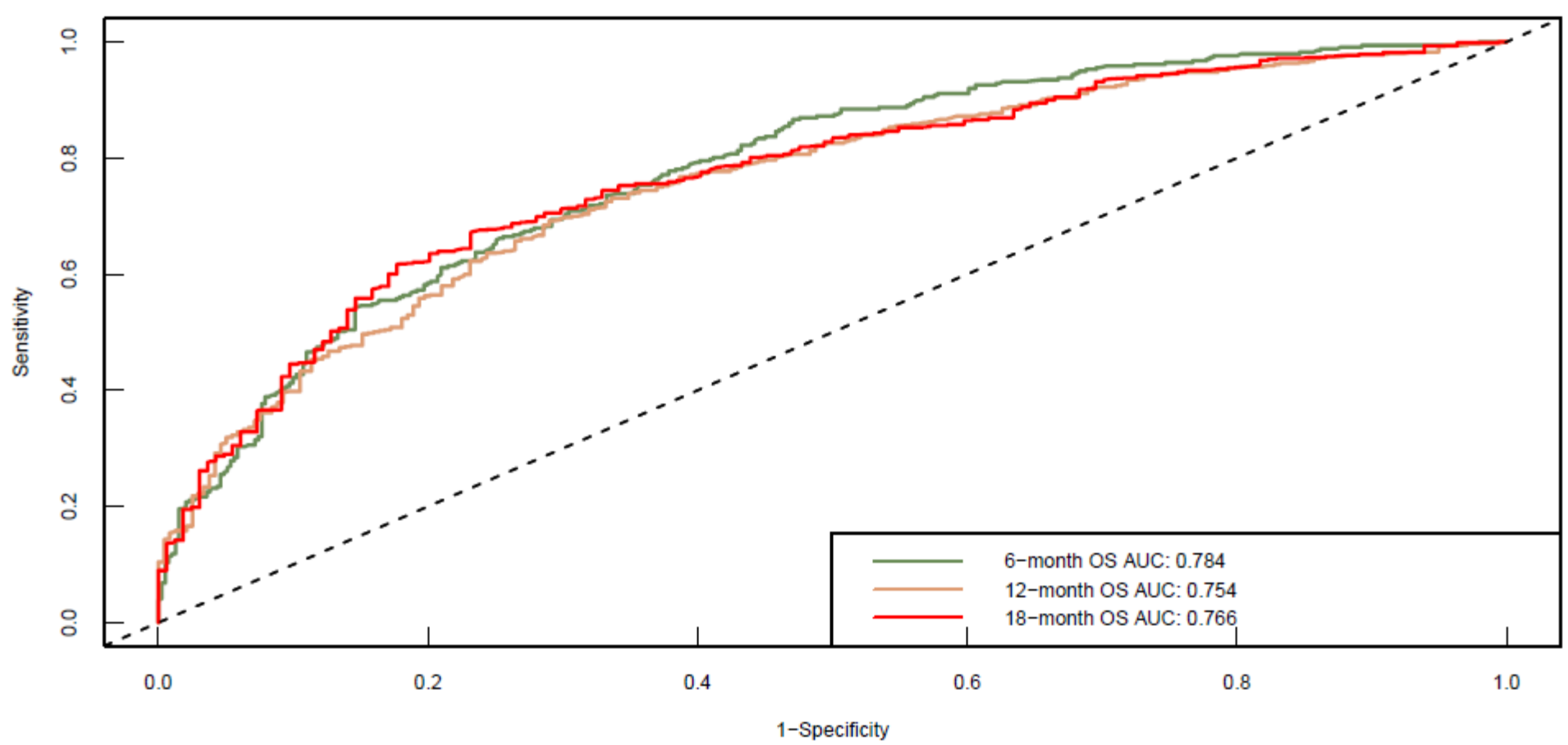

Figure 3

Receiver operating characteristic (ROC) analysis based on nomograms. 
a

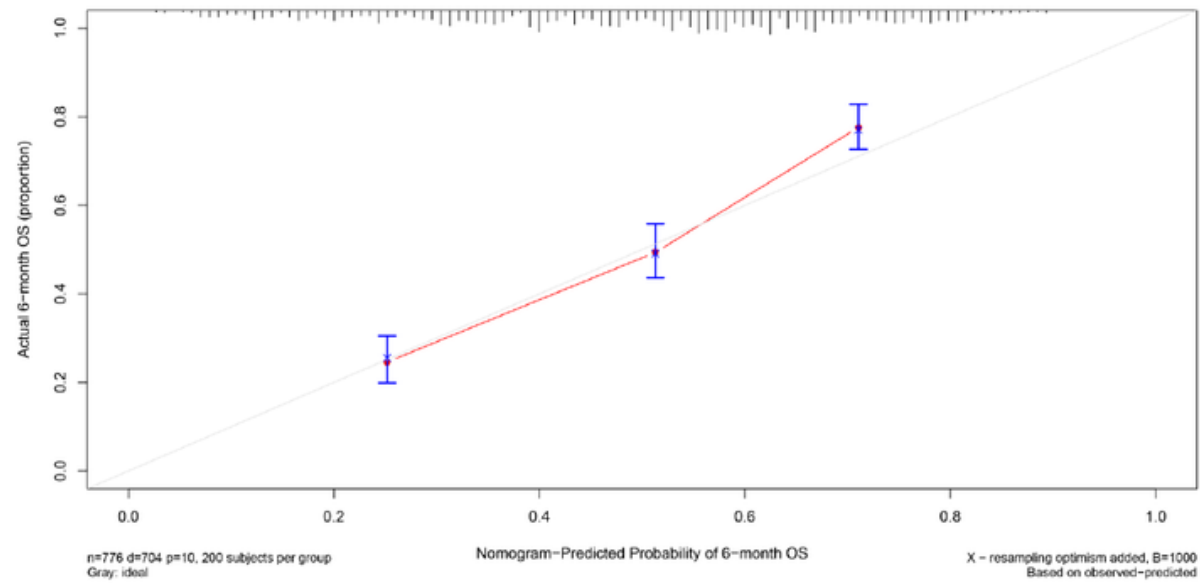

b

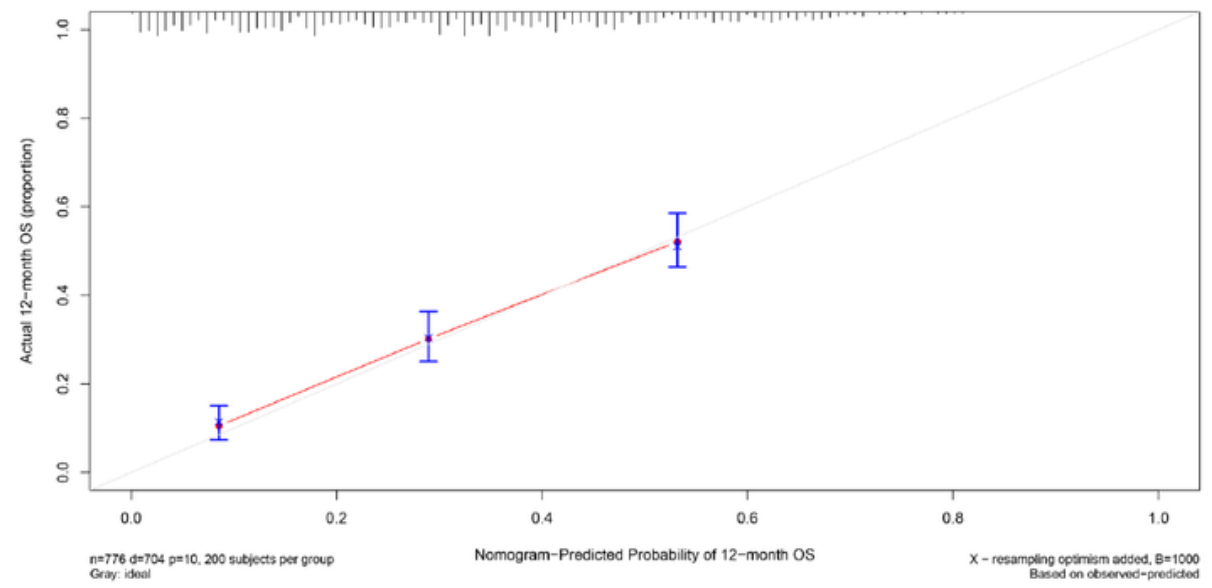

C

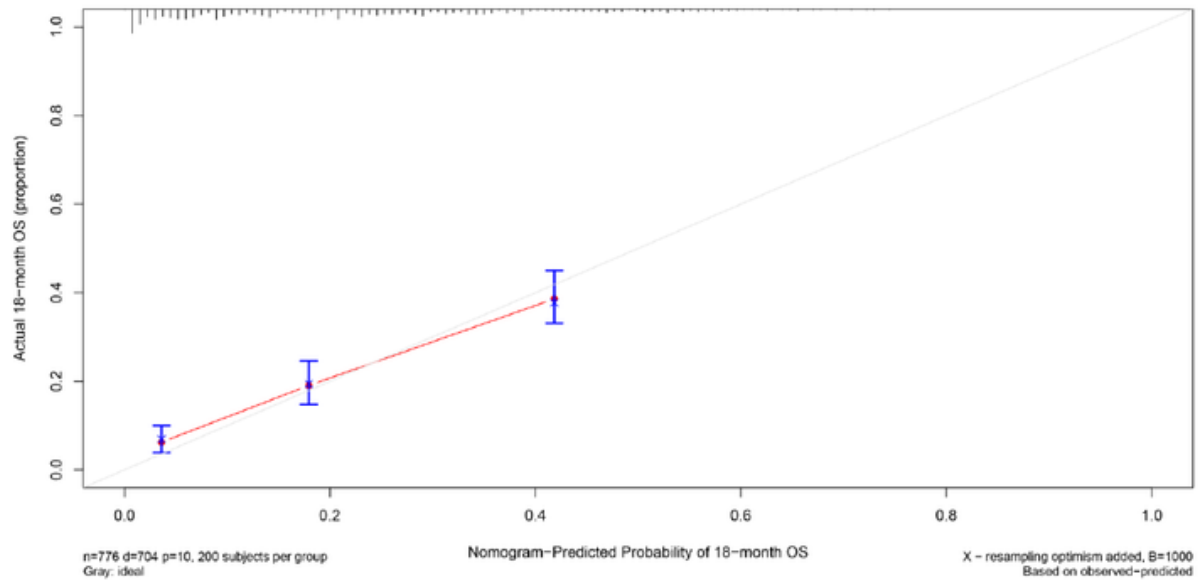

Figure 4

The calibration plots of nomogram for predicting 6-, 12- and 18-month overall survival.

\section{Supplementary Files}

This is a list of supplementary files associated with this preprint. Click to download. 
- SupplementaryFigure1.pdf

- SupplementaryFigure2.pdf 